\title{
Clinical and angioarchitectural characteristics of spinal vascular malformations of the cervical spine
}

\author{
Waleed Brinjikji, MD, ${ }^{1,2}$ Elisa Colombo, MD, ${ }^{1}$ and Giuseppe Lanzino, MD ${ }^{1,2}$ \\ Departments of ${ }^{1}$ Neurosurgery and ${ }^{2}$ Radiology, Mayo Clinic, Rochester, Minnesota
}

\begin{abstract}
OBJECTIVE Vascular malformations of the cervical spine are exceedingly rare. To date there have been no large case series describing the clinical presentation and angioarchitectural characteristics of cervical spine vascular malformations. The authors report their institutional case series on cervical spine vascular malformations diagnosed and treated at their institution.
\end{abstract}

METHODS The authors retrospectively reviewed all patients with spinal vascular malformations from their institution from January 2001 to December 2018. Patients with vascular malformations of the cervical spine were included. Lesions were characterized by their angioarchitectural characteristics by an interventional neuroradiologist and endovascular neurosurgeon. Data were collected on clinical presentation, imaging findings, treatment outcomes, and long-term followup. Descriptive statistics are reported.

RESULTS Of a total of 213 patients with spinal vascular malformations, $27(12.7 \%)$ had vascular malformations in the cervical spine. The mean patient age was $46.1 \pm 21.9$ years and $16(59.3 \%)$ were male. The most common presentations were lower-extremity weakness (13 patients, $48.1 \%$ ), tetraparesis (8 patients, $29.6 \%$ ), and lower-extremity sensory dysfunction (7 patients, $25.9 \%$ ). Nine patients (33.3\%) presented with hemorrhage. Fifteen patients (55.6\%) had modified Rankin Scale scores of $0-2$ at the time of diagnosis. Regarding angioarchitectural characteristics, 8 patients $(29.6 \%)$ had intramedullary arteriovenous malformations (AVMs), $5(18.5 \%$ ) had epidural arteriovenous fistulas (AVFs), 4 (14.8\%) had paraspinal fistulas, 4 (14.8\%) had mixed epidural/intradural fistulas, $3(11.1 \%)$ had perimedullary AVMs, $2(7.4 \%)$ had dural fistulas, and 1 patient (3.7\%) had a perimedullary AVF.

CONCLUSIONS This retrospective study of 27 patients with cervical spine vascular malformations is the largest series to date on these lesions. The authors found substantial angioarchitectural heterogeneity with the most common types being intramedullary AVMs followed by epidural AVFs, paraspinal fistulas, and mixed intradural/extradural fistulas. Angioarchitecture dictated the clinical presentation as intradural shunts were more likely to present with hemorrhage and acute onset myelopathy, while dural and extradural shunts presented as either incidental lesions or gradually progressive congestive myelopathy.

https://thejns.org/doi/abs/10.3171/2019.11.SPINE19798

KEYWORDS spinal vascular malformation; intramedullary hemorrhage; myelopathy; cervical spine; vascular disorders

$\mathrm{A}$ VAST majority of spinal vascular malformations that are encountered are dural or epidural arteriovenous fistulas (AVFs) located in the thoracic and lumbar regions and present with classic symptoms related to gradually progressive lower-extremity myelopathy with bladder and bowel dysfunction. ${ }^{2}$ Spinal vascular malformations involving the cervical spine are exceedingly rare and their clinical and angioarchitectural features have not been described in a comprehensive fashion in the literature to date. ${ }^{8}$ On review of the literature, it is apparent that there is substantial heterogeneity in the angioarchitecture and clinical presentation of cervical spine vascular malforma- tions. Given the rarity of these lesions and the lack of any comprehensive studies or large case series on this topic, we reviewed our institutional case series of patients with vascular malformations of the cervical spine with an emphasis on imaging, clinical presentation, and outcomes.

\section{Methods \\ Patient Population}

Following IRB approval, we identified all patients who had been evaluated and/or treated for a spinal vascular malformation at our institution from January 1, 2001, to

ABBREVIATIONS AVF = arteriovenous fistula; $A$ VM = arteriovenous malformation; DAVF = dural AVF; ICG = indocyanine green; MRA = MR angiography; mRS = modified Rankin Scale; PVA = polyvinyl alcohol; SAH = subarachnoid hemorrhage.

SUBMITTED July 5, 2019. ACCEPTED November 11, 2019.

INCLUDE WHEN CITING Published online January 17, 2020; DOI: 10.3171/2019.11.SPINE19798. 
December 31, 2018. Patients were included if they had a definite diagnosis of a spinal vascular malformation based on spinal angiography or, less often, MR angiography (MRA). After identification of this patient population, we identified the subset of patients who had spinal vascular malformations in the cervical spine region for further review. Patients with dural fistulas of the cervicomedullary junction (i.e., marginal sinus, hypoglossal canal, etc.) were excluded.

\section{Data Collection}

A retrospective chart review was performed. Baseline demographic data collected included age and sex. Presenting symptoms collected included back pain, extremity weakness, urinary dysfunction, bowel dysfunction, and somatosensory dysfunction. Modified Rankin Scale (mRS) scores at the time of presentation, time of diagnosis, and last follow-up were collected as well. We also collected data regarding how each lesion was managed (conservative, surgery, endovascular, etc.) and outcomes of intervention when performed.

\section{Imaging Data}

All imaging was reviewed by an interventional neuroradiologist and an endovascular neurosurgeon (W.B. and G.L.). On MRI, notation was made of cord enhancement, T2 hyperintensity, flow voids, hematomyelia, syrinx, and mass effect from vascular structures. Spinal angiography including conventional angiography, CT angiography, and MRA was reviewed to characterize the angioarchitecture of the lesions including number and location of feeding arteries, location of the shunt, presence of intradural venous drainage, and presence of mass effect from vascular structures.

\section{Classification of Angioarchitecture}

Lesions were classified according to our institutional classification system as follows. A dural arteriovenous fistula (DAVF) was a fistula within the nerve root sleeve dura fed by a radiculomeningeal artery with retrograde drainage into radicular veins toward the perimedullary veins. An epidural AVF was a fistula within the lateral or ventral epidural space between arteries of the epidural arterial arcade and the epidural venous plexus with or without intradural venous drainage. A paraspinal AVF was considered a fistula in the paraspinal soft tissues with venous drainage into epidural or paraspinal veins. An intramedullary arteriovenous malformation (AVM) was a nidus-type AVM located within the cord parenchyma fed by anterior or posterior spinal arteries with drainage into medullary and/or pial veins. A perimedullary AVM was considered a nidus-type AVM located on the surface of the cord with no parenchymal component fed by anterior or posterior spinal arteries with drainage into medullary or pial veins. Perimedullary AVFs were direct fistulas between a hypertrophied spinal artery and hypertrophied spinal vein on the surface of the spinal cord. And lastly, mixed malformations were vascular malformations in which there are shunts both in the epidural space and intradurally. None of our cases of spinal metameric AVMs involved the cervical spine.

\section{Treatment Characteristics and Results}

For lesions that underwent treatment we report the modality of treatment (surgical vs endovascular), embolic agent used (if endovascular), treatment-related complications, and treatment-related outcomes.

\section{Statistical Analysis}

No formal statistical comparisons were performed as part of this study. We report baseline characteristics, imaging findings, and outcomes for the entire patient population and further stratify outcomes based on the classification of the spinal vascular malformation. Categorical variables are presented as value (percentage) and continuous variables as mean (standard deviation). All analyses were performed with the SAS-based statistical software package JMP (version 13.0; www.jmp.com).

\section{Results}

\section{Patient Population}

Of a total of 213 patients with spinal vascular malformations, 27 (12.7\%) had vascular malformations in the cervical spine. The mean patient age was $46.1 \pm 21.9$ years and 16 patients $(59.3 \%)$ were male. The most common presentations were lower-extremity weakness (13 patients, $48.1 \%$ ), tetraparesis (8 patients, $29.6 \%$ ) and lower-extremity sensory dysfunction (7 patients, $25.9 \%$ ). Nine patients $(33.3 \%)$ presented with hemorrhage. Fifteen patients (55.6\%) had mRS scores of 0-2 at the time of diagnosis. Regarding angioarchitectural characteristics, 8 patients (29.6\%) had intramedullary AVMs, 5 (18.5\%) had epidural fistulas, $4(14.8 \%)$ had paraspinal fistulas, $4(14.8 \%)$ had mixed epidural/intradural fistulas, 3 (11.1\%) had perimedullary AVMs, 2 (7.4\%) had dural fistulas, and 1 patient (3.7\%) had a perimedullary AVF. Details regarding each type of vascular malformation are below.

\section{Cervical Spine Dural Fistulas}

Two patients in our series had cervical spine DAVFs. The mean patient age was $62 \pm 14.1$ years. Both patients were male and presented with lower-extremity weakness. No patients had upper-extremity weakness or hemorrhage. High cord signal intensity and enhancement was present in 1 patient extending to the conus. Both patients had flow voids on MRI. Neither patient had a syrinx. One patient was successfully treated surgically with a $\mathrm{C} 1$ laminectomy and surgical disconnection of the arterial-venous connection at $\mathrm{C} 1$. Postoperative MRI showed reduction in spinal cord edema and posterior cervical spine flow voids at 1 month and 4 years after surgical disconnection. Clinically the patient had a dramatic improvement in lower-extremity weakness. The other patient elected not to undergo treatment. An example of a cervical spine DAVF is provided in Fig. 1.

\section{Cervical Spine Epidural AVFs}

Five patients in our series had cervical epidural AVFs. An example of a cervical spine epidural AVF is provided in Fig. 2. The mean patient age in this group was 42.0 \pm 21.7 years. One patient $(20.0 \%)$ was male. One pa- 

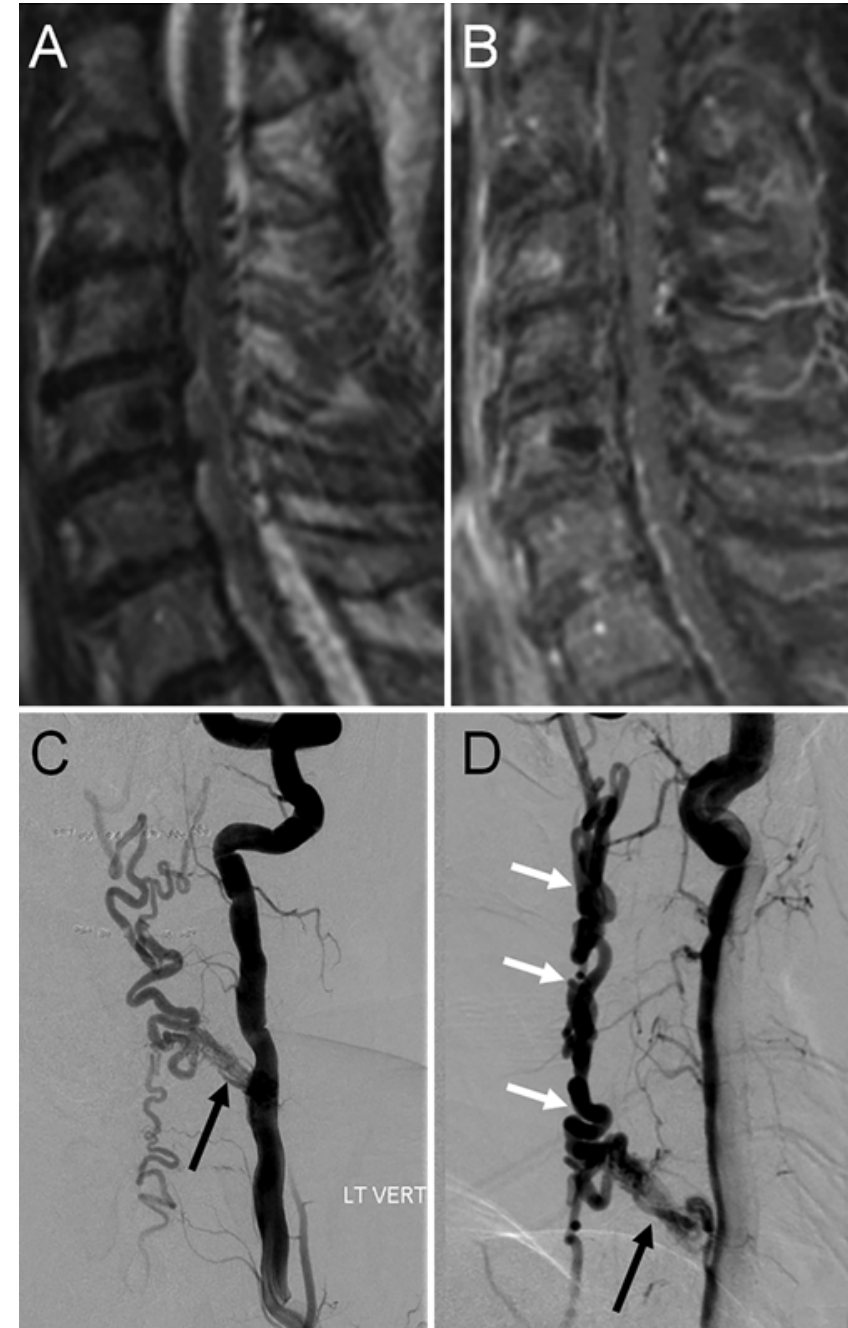

FIG. 1. Example of a cervical spine DAVF. Images obtained in a 72-yearold man with a history of progressive ataxia secondary to a cervical spine DAVF. A: Sagittal T2-weighted MR image shows multiple perimedullary flow voids along the posterior aspect of the cord. B: Sagittal T1-weighted postcontrast MR image shows enhancement of the flow voids but no spinal cord enhancement. C: Anteroposterior left vertebral artery cervical angiogram shows a DAVF along the left C5 nerve root fed by a radicular branch of the left vertebral artery (arrow). D: Lateral left vertebral artery angiogram shows the AVF (black arrow) with filling of engorged veins along the dorsal aspect of the spinal cord (white arrows). This lesion was managed conservatively as the patient opted for no treatment following an unrelated left middle cerebral artery stroke.

tient $(20.0 \%)$ presented with tetraparesis. Three lesions were found incidentally and one during angiography for perimesencephalic hemorrhage. These incidental lesions were not treated as they did not have any intradural venous drainage.

One lesion presented with mass effect and quadriparesis from a large venous varix. This lesion was treated in 2005 with preoperative embolization using 250- to 300$\mu \mathrm{m}$ polyvinyl alcohol (PVA) particles through the thyrocervical trunk and was subsequently treated surgically with laminectomies and facetectomies at $\mathrm{C} 4-7$ and surgical excision of the entirety of the malformation, followed by $\mathrm{C} 3-\mathrm{T} 1$ posterior fusion. This patient experienced grad-
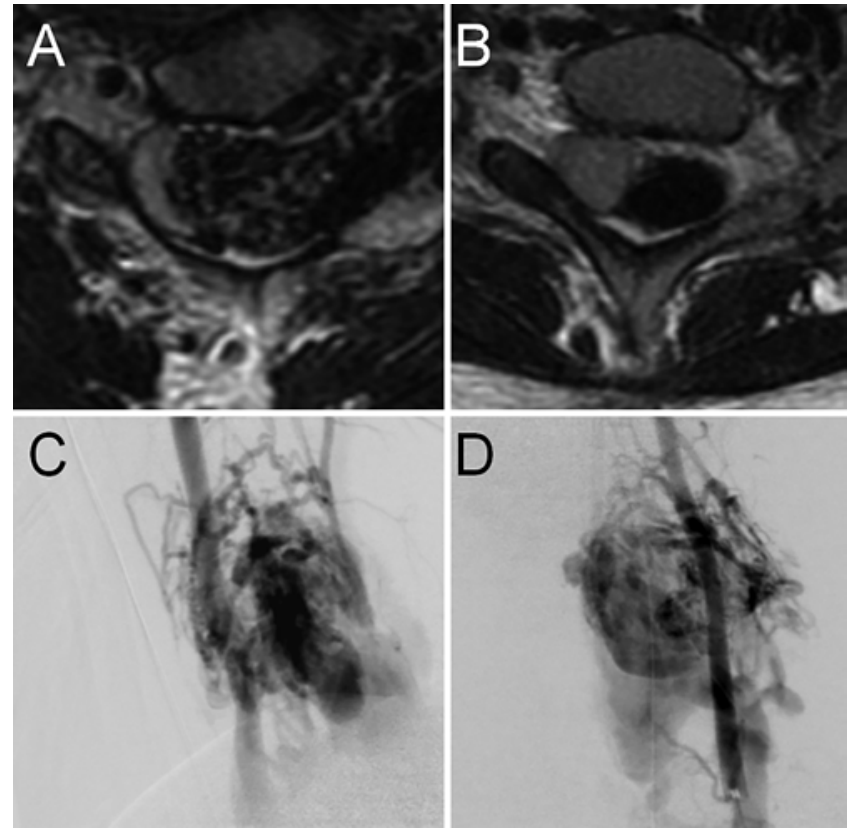

FIG. 2. Example of a cervical spine epidural AVF in a 13-year-old girl with weakness and spasticity involving both upper and lower extremities bilaterally, and frequent falls. Symptoms progressed over 5 months. A and B: Axial T2-weighted MR images show prominent intradural flow voids that are compressing the cervical spine cord. There is some elevated T2 signal of the cord in panel A. C and D: Anteroposterior and lateral left vertebral artery angiograms show an extensive epidural AVF involving the spinal cord and the surrounding tissues extending from $\mathrm{C} 4$ to $\mathrm{T} 1$. Note the markedly enlarged extradural varix compressing the spinal cord. There was no intradural venous drainage. The patient underwent staged embolization with PVA particles and resection of the shunt, with immediate improvement. At follow-up, serial MR tests showed interval resolution of the cord changes and the patient regained full functionality (not shown).

ual resolution of her quadriparesis and remains ambulatory and functionally independent 14 years later.

\section{Cervical Spine Intramedullary AVMs}

Eight patients in our series had intramedullary AVMs, with a mean patient age of $39.5 \pm 16.0$ years. Four patients $(50.0 \%)$ were male. The most common presentation was hemorrhage (5 patients, $62.5 \%)$. Two patients $(25.0 \%)$ had lower-extremity weakness, one of whom had tetraparesis. On MRI, 3 patients (37.5\%) had increased cord signal, 4 $(50.0 \%)$ had intradural flow voids, and $4(50.0 \%)$ had cord enhancement. Five patients $(62.5 \%)$ received treatment with either resection (1 patient) or endovascular treatment (4 patients). Of the 4 patients treated with endovascular embolization, 3 were treated with palliative embolization using PVA particles. The fourth was treated with coil embolization of a ruptured prenidal aneurysm. The surgically treated patient had complete obliteration of the AVM on follow-up angiography. An example of a cervical spine intramedullary AVM is provided in Fig. 3.

\section{Cervical Spine Perimedullary AVFs}

One patient had a single-hole perimedullary AVF. The patient presented with acute onset quadriparesis with an 


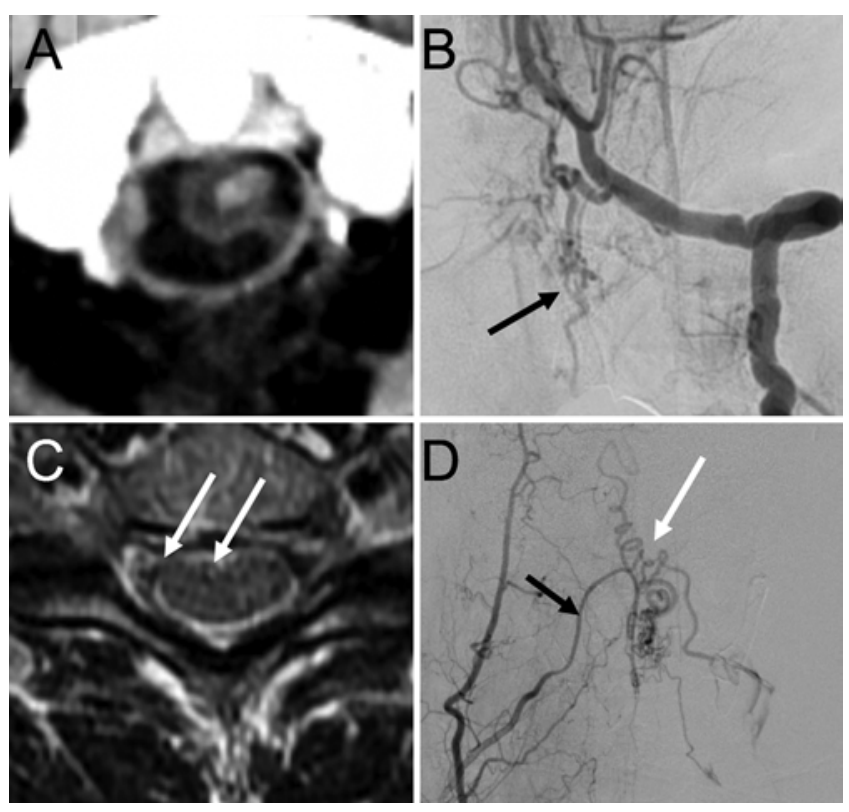

FIG. 3. A and B: Intramedullary spinal cord AVM in a 61-year-old woman transferred in emergency to our institution for "the worst headache ever." Noncontrast CT (A) shows a focus of intramedullary hemorrhage as well as a small amount of SAH. Left vertebral artery angiogram (B) shows an intramedullary AVM with a nidus located at $\mathrm{C} 1$ with drainage along the spinal veins (arrow). The AVM was treated surgically and follow-up catheter angiography confirmed complete obliteration of the fistula (not shown). C and D: Images from a 56-year-old woman with a 15-year history of involuntary quick jerklike movements involving all 4 extremities. Spinal cord axial MRI (C) shows subtle flow voids along the ventral aspect of the cord and in the cord parenchyma itself (arrows). There is also some T2 hyperintensity along the left aspect of the spinal cord. Deep cervical artery spinal angiogram (D) shows an intramedullary spinal AVM at C5-6 fed by branches of the anterior spinal artery (black arrow) draining into dilated and tortuous spinal veins (white arrow). The AVM was managed conservatively. At follow-up, the patient endorsed a mild increase in the frequency of the movements, but the lesion did not show any apparent interval growth on serial imaging.

MRI scan that showed a large cervical AVF with a compressive varix causing spinal cord edema at the $\mathrm{C} 4$ level. The patient underwent endovascular embolization with coils that were placed at the site of the AVF and the fistula was obliterated. The patient partially recovered function in his upper extremities but remained wheelchair bound with neurogenic bladder. Follow-up MRI showed resolution of the fistula. Representative images are provided in Fig. 4.

\section{Cervical Spine Paraspinal Fistulas}

Four patients had paraspinal fistulas of the cervical spine. The mean age of this group was $38.5 \pm 26.1$ years. Three patients (75.0\%) were male. Two patients had upperextremity weakness and sensory changes. There were no spinal cord changes in any of the patients. No patients had hemorrhage. One patient was treated surgically with surgical disconnection of the arteriovenous connection and resection of the AVM in the paraspinal tissues. Three patients were treated endovascularly. Of the 3 patients treated endovascularly, one was treated with coil embolization of the fistula along with PVA injection, and the other two were treated with particle embolization for palliative purposes. On follow-up angiography, two were completely obliterated and two partially obliterated. The lesions treated with particle embolization were only partially obliterated. Representative images are shown in Fig. 5.

\section{Cervical Spine Mixed Intradural and Extradural AVFs}

Four patients had mixed intradural and extradural fistulas, with a mean patient age of $70.3 \pm 10.9$ years. Two patients $(50.0 \%)$ were male. Two patients presented with lower-extremity weakness and sensory changes. One patient presented with tetraparesis. Three patients had spinal hemorrhage, one subarachnoid (SAH) and two intramedullary. On MRI, 2 patients had high cord signal intensity, flow voids, and cord enhancement. Three patients underwent treatment (one surgical and two endovascular) and follow-up angiography showed some filling of the fistula in the 2 endovascularly treated patients. The endovascularly treated patients were treated with coil embolization of the ruptured prenidal aneurysm. Representative images are shown in Fig. 6.

\section{Perimedullary AVMs}

Three patients had perimedullary AVMs. Representative images are shown in Fig. 7. The mean patient age was $47.7 \pm 30.1$ years. All 3 patients were male. Two patients presented with tetraparesis. All 3 patients presented with sensory changes, urinary dysfunction, and bowel dysfunction. No patients had hemorrhage. Two patients had T2 signal changes and enhancement of the cord. All 3 patients had flow voids. One patient was treated surgically and one with embolization and surgery.

The surgically treated patient was treated with laminectomies from $\mathrm{C} 3$ to $\mathrm{C} 5$ followed by a durotomy. Intraoperatively there was dilation of a posterior spinal artery and the posterior spinal venous plexus. The site of the arteriovenous connection was isolated and disconnected. Intraoperative and postoperative angiography showed resolution of the lesion and postoperative MRA 5 years later showed no residual fistula. Postoperative angiography showed obliteration of the surgically treated lesion and persistent filling of the endovascularly treated lesion.

The other patient was treated with attempted Onyx (Medtronic) embolization at an outside center, which was complicated by a perforation of the feeding posterior spinal artery and reflux into the vertebral artery. Repeat angiography at our center showed a residual fistula. The patient was taken for resection including removal of the posterior arch of $\mathrm{C} 1$ and a suboccipital craniectomy. The intradural arterialized vein was identified and a permanent clip was placed across the vein. Complete occlusion was confirmed with indocyanine green (ICG) fluorescein angiography and subsequent spinal angiography. Twelve-month postoperative MRI showed resolution of cervical spine signal changes and flow voids and the patient has experienced a dramatic improvement in neurological symptoms.

The final patient is highlighted in Fig. 7C and D. When performing superselective angiography we identified a radiculomedullary artery arising from the same pedicle supplying the posterior spinal artery that supplied the fistula. 


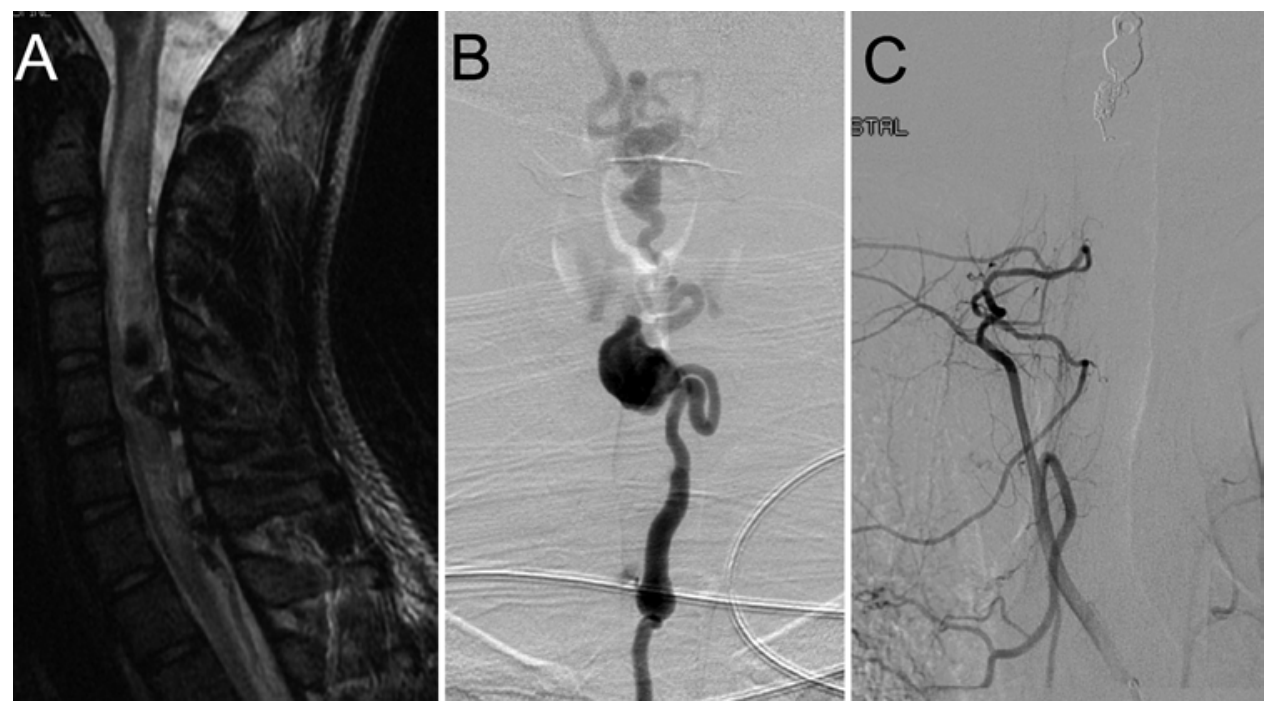

FIG. 4. Single-hole perimedullary AVF in a 17-year-old boy presenting to the emergency department with sudden-onset neck and right-shoulder pain followed by flaccid quadriparesis. On examination, he had a sensory level at C4 for all modalities with absent reflexes. A: Sagittal T2-weighted MRI shows dilated vessels along the dorsal aspect of the spinal cord and evidence of high cord signal intensity as well as a focus of T2 hypointensity consistent with hematomyelia at C5. B: Spinal angiography with injection of the right supreme intercostal artery shows a hypertrophied posterior spinal artery connecting with a dilated venous varix and then draining into spinal veins along the posterior aspect of the cord. Urgent coil embolization was performed. The patient showed mild symptomatic improvement but still suffered from significant spasticity. C: Follow-up angiography showed resolution of the AVF.

For this reason, we proceeded with resection. The patient underwent a C6-T1 laminoplasty. Following identification of the largest feeder, a temporary clip was placed on the artery. Low-power bipolar cautery was used to progressively shrink, coagulate, and separate the AVM nidus from the pial surface and the C6/7 nerve roots. After ICG fluorescein confirmation that the nidus was coagulated, the draining vein was coagulated and divided. Postoperative angiography and ICG fluorescein showed no residual filling.

\section{Discussion}

Our retrospective review of cervical spinal vascular malformations evaluated at our institution is the largest series to date on this topic. We found substantial heterogeneity in the angioarchitectural features and clinical presentation of patients with cervical spine vascular malformations. Unlike spinal vascular malformations located in the thoracolumbar regions, the most common type of fistulas seen among patients with cervical spine lesions were intramedullary AVMs followed by purely epidural and mixed intradural/epidural lesions. Interestingly, 16 of 27 patients with cervical spine vascular malformations had an intradural shunt. Dural fistulas were very rare. Of patients with intradural shunts, $50 \%$ presented with either SAH or intramedullary hemorrhage. These findings are important as they highlight the diverse angioarchitecture of cervical spine vascular malformations and the association between angioarchitecture and clinical symptoms.

There have been many case reports and small case series detailing the clinical presentation, angiographic features, and outcomes of patients with cervical spine vascular malformations. These reports also highlight the substantial heterogeneity in the angioarchitectural features of cervical spine vascular malformations. In our review of the literature (Supplemental Tables 1-7) we found a total of 204 cervical spine vascular malformations. Of these, 40 (19.6\%) were spinal DAVFs, 28 (13.7\%) were spinal epidural AVFs, $52(25.5 \%)$ were intramedullary AVMs, 17 (8.3\%) were perimedullary AVMs/AVFs, 16 (7.8\%) were paraspinal AVFs, and 7 (3.4\%) were combined extradural/ intradural AVFs. However, to date, there has been no large cross-sectional analysis of the features of cervical spine vascular malformations as seen in our case series. The distribution of spinal vascular malformations of the cervical spine noted in the literature is roughly similar to that seen in our institutional case series, with intramedullary AVMs being the most common lesions. Hemorrhage was present in roughly one-third of the cases in our series compared to approximately $25 \%$ of patients in the literature.

\section{Cervical Spine DAVFs}

Dural fistulas of the cervical spine are exceedingly rare and comprise less than $1 \%$ of lesions in large institutional case series of spinal DAVFs. ${ }^{5}$ These lesions are distinct from dural fistulas of the craniocervical junction as they occur in the nerve root sleeve of the cervical nerve roots rather than in the dura of the skull base. Angioarchitecturally they are composed of a radiculomeningeal artery feeder anastomosing with a radiculomedullary vein. Similar to thoracolumbar lesions, cervical dural fistulas present with gradual onset myelopathy with $\mathrm{T} 2$ changes ascending from the conus. Endovascular treatment of these lesions can be difficult due to the fact that the feeding radicular artery arises from the vertebral artery, thus rendering the risk of reflux too high. The reason why cervical spine DAVFs are so rare is unclear. 


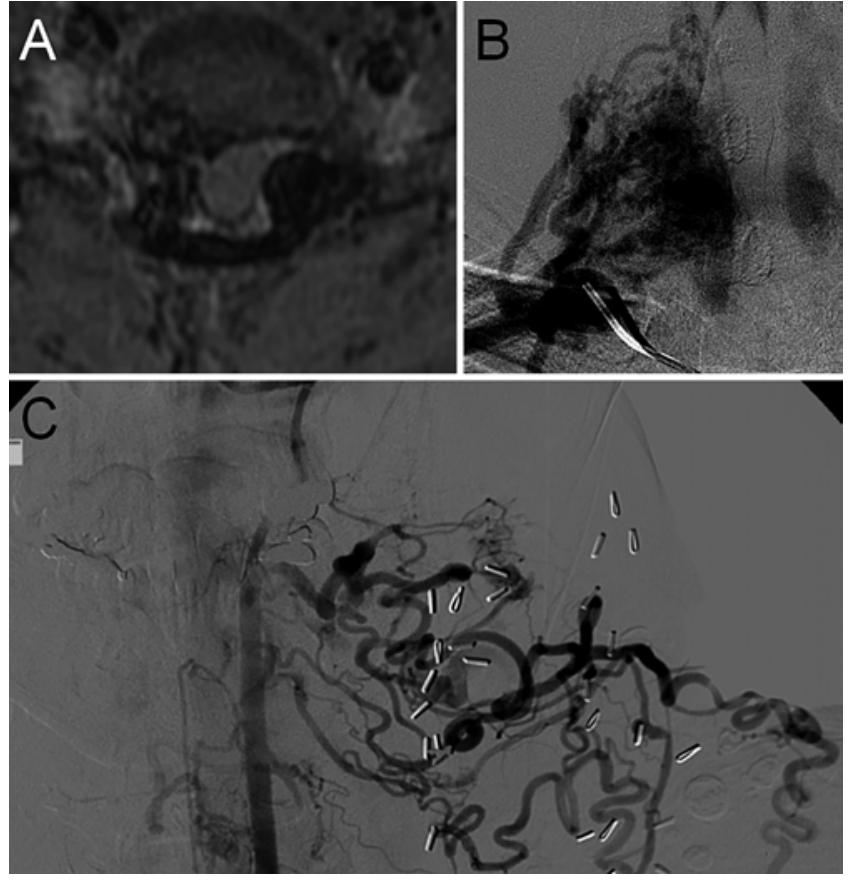

FIG. 5. Paraspinal cervical AVFs. A and B: Images from a 12-year-old girl with a history of subacute sensory and motor changes in the four limbs. Axial T2-weighted MRI (A) shows multiple flow voids in the right paraspinal soft tissues. There is also engorgement of the epidural venous plexus. There is high T2 signal in the cord, likely due to a combination of arterial steal and compressive myelopathy. A catheter angiogram (B) shows an extensive AVM/AVF in the paraspinal soft tissues with extradural venous drainage. A partial embolization was performed with particles. At 1-year follow-up the patient showed good recovery, and MRI of the cervical cord documented progressive resolution of the abnormal changes. C: Angiographic image obtained in a 44-year-old man with a long history of anterior neck pain leading to the discovery of a complex cervical AVM involving the left shoulder. The lesion was embolized and surgical treatment was attempted at an outside center. The patient presented at our institution for new-onset posterior neck pain. Radiological evaluation showed interval increase of the lesion with recruitment of new arterial feeders. Particle embolization of the largest part of the AVM was performed successfully, with immediate pain relief. Follow-up MRI studies showed interval changes of the residual AVM with remodeling of the ipsilateral hypoglossal canal (not shown). The AVM was managed conservatively.

\section{Cervical Spine Epidural Fistulas}

Epidural fistulas of the cervical spine are typically located within the lateral or ventral epidural space. ${ }^{1}$ Angioarchitecturally they are characterized by anastomoses between arteries of the epidural arterial arcade and veins of the epidural venous plexus. The symptomatology of these lesions is dependent on the angioarchitecture. Lesions with intradural venous drainage will present with a congestive myelopathy similar to that seen in patients with spinal dural fistulas. Meanwhile, patients with large fistulas associated with venous engorgement can present with a compressive myelopathy due to compression of the cord by the venous varix. Lesions located in the epidural space without venous engorgement or intradural venous drainage are typically asymptomatic and managed conservatively. Management is highly dependent on angioarchitecture and both surgical and endovascular therapies have been used with varying levels of success. However, recently there has been a trend toward more of these lesions being treated endovascularly due to the fact that liquid embolic agents can be used to penetrate the entirety of the epidural venous pouch.

\section{Cervical Spine Intramedullary AVMs}

Intramedullary AVMs are nidus-type AVMs that are located in the parenchyma of the spinal cord. Angioarchitecturally, these lesions are similar to AVMs of the brain. ${ }^{9}$ They can be associated with feeding arterial and intranidal aneurysms and have various routes of venous egress with venous ectasia, stenosis, and congestion. A majority of these lesions in both the literature and our series present with hemorrhage, usually due to rupture of an intranidal aneurysm. The remainder of these lesions present with a focal myelopathy secondary to either local venous congestion or local arterial steal phenomenon. While the majority of cases in the literature have been treated surgically, there has been a trend toward more and more cases being treated endovascularly in recent times. Regarding endovascular treatment, targeted treatment of angiographic weak points (i.e., prenidal or intranidal aneurysms) with glue or coils is the most commonly used treatment technique. Some centers have opted to treat cervical spine AVMs palliatively with particle embolization while others have attempted to cure these lesions with glue embolization.

\section{Cervical Spine Perimedullary AVFs}

Single-hole or multihole perimedullary AVFs are angioarchitecturally similar to direct pial AVFs in the brain. These lesions are commonly observed in patients with HHT and RASAl mutations. ${ }^{4}$ These lesions are characterized by a markedly hypertrophied spinal artery connecting directly to an even more hypertrophied spinal vein. Symptomatically, these lesions can present with congestive myelopathy from venous congestion, compressive myelopathy related to engorgement and/or thrombosis of a venous varix, or sudden-onset myelopathy from rupture of either a feeding arterial aneurysm or venous varix. Endovascular intervention is typically favored for treatment of these lesions as it is generally easy to catheterize the markedly hypertrophied artery and disconnect the arteriovenous connection using glue or coils.

\section{Cervical Spine Paraspinal Fistulas}

Angioarchitecturally, paraspinal AVFs are characterized as direct fistulas in the paraspinal soft tissues with venous drainage into epidural or paraspinal veins. ${ }^{10}$ These lesions are relatively rare with only 16 cases reported in the literature. Paraspinal AVFs generally present with progressive neurological symptoms, usually due to arterial steal phenomenon from the spinal cord itself. Because the fistulas are somewhat remote from the spinal canal itself, there is no intradural venous drainage as there are plenty of veins in the paravertebral soft tissues for them to drain into. The favored treatment modality has been endovascular embolization as it is generally safe and effective and easy to target the arteriovenous connection. 


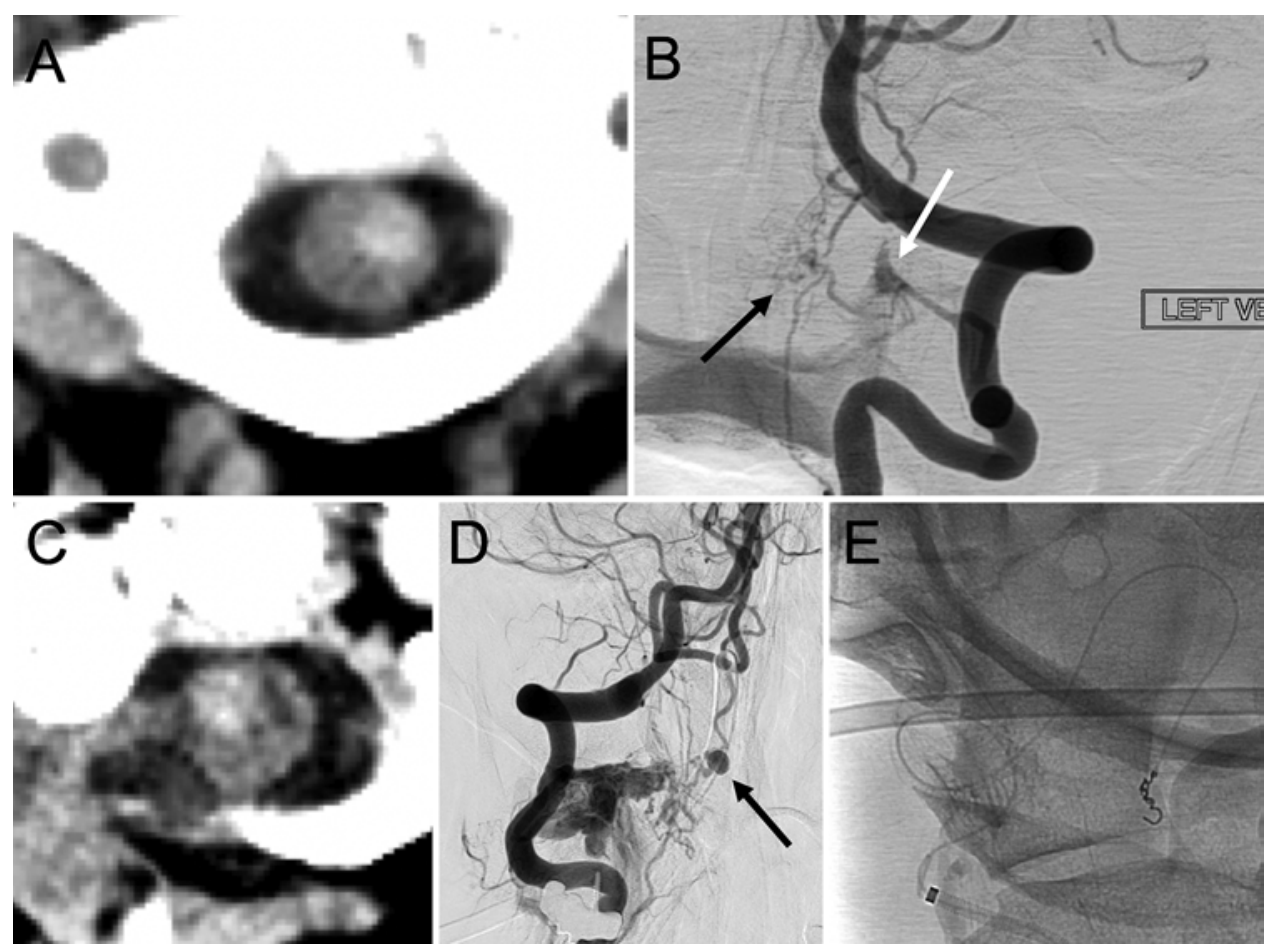

FIG. 6. Mixed intradural and extradural AVFs. A and B: Images obtained in an 81-year-old woman for new-onset headache involving the left parietooccipital region but otherwise she denied any neurological symptoms. Head CT (A) showed a hyperdensity involving the left lateral cervical cord, concerning for hemorrhage. Conventional angiography (B) showed a small pial fistula at the craniocervical junction with both an intradural and extradural component (arrows). Surgical treatment was proposed but refused. The fistula was managed conservatively. C-E: Images in a 56-year-old woman with acute right hemiplegia and right hemisensory loss as well as dysarthria and respiratory depression with need for intubation. Noncontrast CT (C) demonstrated an acute cervical medullary hemorrhage. Catheter angiography (D) showed an epidural AVF fed primarily by the vertebral artery. There was also a small perimedullary shunt along the surface of the cord with a spinal artery aneurysm (arrow). One month after the hemorrhagic event the aneurysm was treated with coil embolization $(E)$. She recovered motor function but suffered from chronic right-sided pain.

\section{Cervical Spine Mixed Intradural and Extradural Fistulas}

Cervical spine mixed intradural and extradural fistulas are exceedingly rare and poorly described in the literature. ${ }^{7}$ These lesions are characterized as vascular malformations in which there are shunts both in the epidural space or dura and intradurally. The pathogenesis of these lesions is unclear. It is possible that the presence of venous hypertension from one fistula could induce the formation of the second fistula. Prior studies have suggested that venous hypertension or increased blood flow in the lowpressure venous system can promote the development of microscopic arteriovenous shunts. ${ }^{3}$ It is also possible that these lesions are due to dysfunction in the development of arteries and veins in a given metamere as the extradural and intradural lesions are usually at the same level. ${ }^{6}$ Again, the presentation of these lesions is highly dependent on the angioarchitecture. In our series, spinal cord hemorrhage was the most common presentation, similar to what has been seen in the literature. Endovascular intervention to treat any angioarchitectural weak points (as seen in Fig. 6) can be performed. Because the intradural shunt is often a single-hole shunt on the surface of the cord, surgical disconnection can be performed as well.

\section{Perimedullary AVMs}

Perimedullary AVMs are distinct from perimedullary
AVFs and intramedullary AVMs, as they are nidus-type AVMs located on the surface of the cord rather than in the cord parenchyma itself. These lesions typically present with congestive myelopathy as they are slower flow than perimedullary AVFs. On imaging they can have a very similar appearance to spinal dural fistulas with flow voids along the surface of the cord. It is not until conventional spinal angiography is performed that one realizes that the feeding arteries to these lesions are spinal arteries rather than radicular arteries. These lesions can be safely embolized or disconnected surgically.

\section{Limitations}

Our study has limitations. First, our institution is a large referral institution for patients with spinal vascular malformations so there may be referral bias that could impact the distribution of the types of spinal vascular malformations seen in our series. Second, our study is retrospective and may be prone to biases related to the study design and inconsistencies in imaging protocols, clinical evaluation, and follow-up duration. It is difficult to draw conclusions about the types of lesions seen in the cervical spine from such a small single-institution sample. Imaging and treatments have changed considerably over the study period. Nonetheless, our study highlights some of the salient clinical, anatomical, imaging, and angiographic findings 

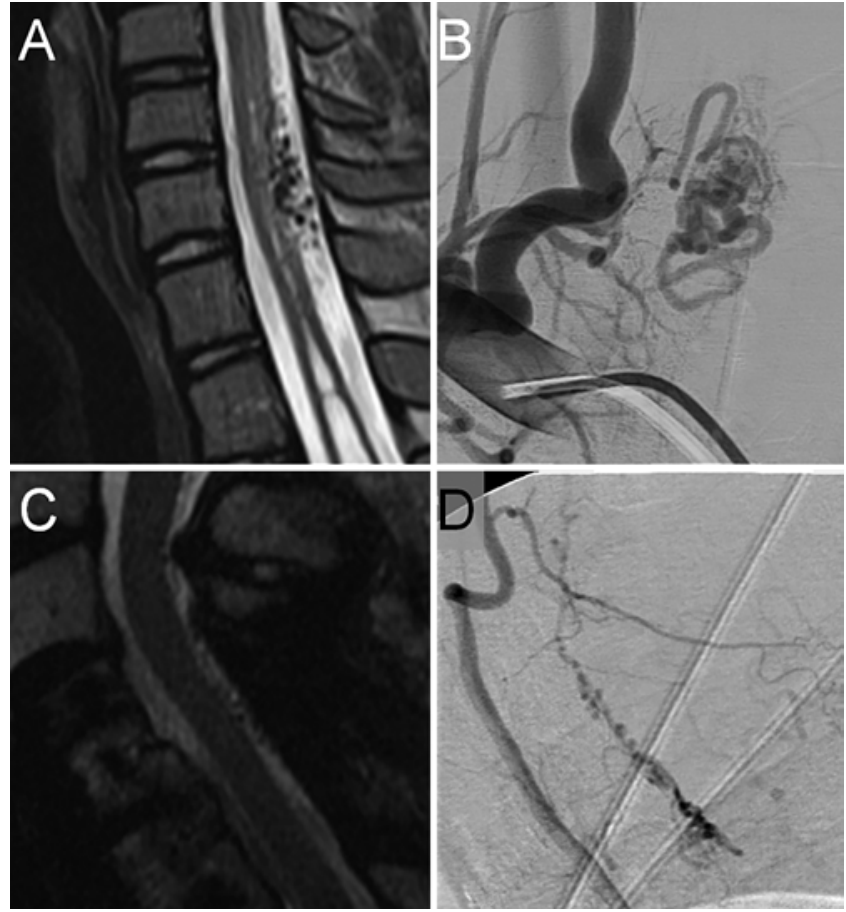

FIG. 7. Cervical perimedullary AVMs. A and B: Images from a 14-yearold boy with a complex history of vertebral dysgenesis, severe thoracolumbar spinal cord myelomalacia and atrophy, tethering of the cord, and syringomyelia since birth. Sagittal T2-weighted MRI demonstrated flow voids along the posterior surface of the spinal cord (A). Conventional angiography (B) demonstrated a cervical perimedullary AVM predominantly fed by hypertrophic posterior spinal arteries. C and D: Images from a 72-year-old man with a history of progressive upper-extremity weakness over 5 years. Sagittal T2-weighted MRI (C) shows multiple flow voids along the dorsal aspect of the cord. The patient underwent multiple embolization attempts at another institution. Spinal angiography at our institution (D) shows a perimedullary AVM along the surface of the cord. The patient was brought to the operating room and an intradural abnormal vessel at $\mathrm{C} 4$ was cauterized. Postoperative catheter angiography showed no residual filling of the fistula (not shown).

among a heterogeneous group of patients with spinal vascular malformations of the cervical spine.

\section{Conclusions}

Our retrospective study of 27 patients with cervical spine vascular malformations is the largest series to date on these lesions. We found substantial angioarchitectural heterogeneity with the most common types being intramedullary AVMs followed by epidural AVFs, paraspinal fistulas, and mixed intradural/extradural fistulas. Angioarchitecture dictated the clinical presentation as intradural shunts were more likely to present with hemorrhage and acute onset myelopathy, while dural and extradural shunts presented as either incidental lesions or gradually progressive congestive myelopathy. Further studies are needed to better understand the pathophysiology and treatment outcomes for these lesions.

\section{References}

1. Brinjikji W, Yin R, Nasr DM, Lanzino G: Spinal epidural arteriovenous fistulas. J Neurointerv Surg 8:1305-1310, 2016

2. Goyal A, Cesare J, Lu VM, Alvi MA, Kerezoudis P, Brinjikji W, et al: Outcomes following surgical versus endovascular treatment of spinal dural arteriovenous fistula: a systematic review and meta-analysis. J Neurol Neurosurg Psychiatry 90:1139-1146, 2019

3. Lai CW, Agid R, van den Berg R, Ter Brugge K: Cerebral arteriovenous fistulas induced by dural arteriovenous shunts. AJNR Am J Neuroradiol 26:1259-1262, 2005

4. McGurgan IJ, Lonergan R, Killeen R, McGuigan C: Cervical spine arteriovenous fistula associated with hereditary haemorrhagic telangiectasia. BMJ Case Rep 2017:bcr2016218172, 2017

5. Richard SA, Ma L, Li H, Li J, You C: Giant intradural cervical spine arteriovenous malformations - a case and review of literature. Neurol Neurochir Pol 52:528-533, 2018

6. Rodesch G, Hurth M, Alvarez H, Ducot B, Tadie M, Lasjaunias P: Angio-architecture of spinal cord arteriovenous shunts at presentation. Clinical correlations in adults and children. The Bicêtre experience on 155 consecutive patients seen between 1981-1999. Acta Neurochir (Wien) 146:217227, 2004

7. Sato K, Endo T, Niizuma K, Fujimura M, Inoue T, Shimizu $\mathrm{H}$, et al: Concurrent dural and perimedullary arteriovenous fistulas at the craniocervical junction: case series with special reference to angioarchitecture. J Neurosurg 118:451-459, 2013

8. Sträter R, Kurlemann G, Schuierer G, Lasjaunias P, Palm DG: [Arteriovenous malformations of the cervical spinal cord.] Klin Padiatr 209:84-87, 1997 (German)

9. Velat GJ, Chang SW, Abla AA, Albuquerque FC, McDougall CG, Spetzler RF: Microsurgical management of glomus spinal arteriovenous malformations: pial resection technique: Clinical article. J Neurosurg Spine 16:523-531, 2012

10. Wendl CM, Aguilar Pérez M, Felber S, Stroszczynski C, Bäzner H, Henkes H: Paraspinal arteriovenous fistula: Stuttgart classification based on experience and a review of the literature. Br J Radiol 91:20170337, 2018

\section{Disclosures}

The authors report no conflict of interest concerning the materials or methods used in this study or the findings specified in this paper.

\section{Author Contributions}

Conception and design: Brinjikji, Lanzino. Acquisition of data: all authors. Analysis and interpretation of data: Brinjikji, Lanzino. Drafting the article: all authors. Critically revising the article: all authors. Reviewed submitted version of manuscript: all authors. Approved the final version of the manuscript on behalf of all authors: Brinjikji. Statistical analysis: Brinjikji.

\section{Supplemental Information \\ Online-Only Content}

Supplemental material is available with the online version of the article.

Supplemental Tables 1-7. https://thejns.org/doi/suppl/10.3171/ 2019.11.SPINE19798.

\section{Correspondence}

Waleed Brinjikji: Mayo Clinic, Rochester, MN. brinjikji.waleed@ mayo.edu. 